\title{
Guidelines for environmental health surveillance with respect to air pollution related to fires and effects on human health
}

\author{
Luciana Costa ${ }^{1}$, Fábio David ${ }^{2}$, Gustavo Souza ${ }^{3}$
}

\footnotetext{
${ }^{1}$ Technical Consultant - Pan American Health Organization -OPAS;

2 Technical Analyst of Social Policies;

${ }^{3}$ Full technologist - Brazil.
}

\begin{abstract}
The issue of fires in Brazil ranges from environmental damage and losses to regional economys to harmful effects on human health. In much of the country is commonly used in some agricultural practices. Their different kinds of biomass emit various pollutants and quite varying concentrations. The content of the smoke from the fires is not homogeneous, and the health effects are not, as well. The purpose of this paper is to support the state and municipal managers regarding the performance in environmental health surveillance activities for air pollution related to fires and effects on human health. Air pollution is a major environmental health problem currently, being associated with various deleterious effects on human health. According to the Unified Health System (SUS), the degradation of air quality directly affects costs and overloads the public health system, representing an increase in emergency care and hospital admissions for respiratory and cardiovascular diseases. Circulatory diseases were the leading cause of death $(27.6 \%)$ in the country in 2016, while respiratory diseases were the third leading cause of hospitalization $(10,3 \%)$ and the third leading cause of death $(12,1 \%)$ in 2017 . Since part of the total hospitalization and deaths might be attributed to exposure to smoke from biomass burning, fire prevention measures and integrated fire management are required to improve health statistics, as well as reducing the demand and burden of SUS. The Draft Law of the National Integrated Fire Management Policy foresees the involvment of diverse social players, based on the strengthening of intra and interinstitutional joint. Thus, environmental health surveillance encourages actions capable of diminishing impacts of fires on human health aimed at the well-being and health protection, considering to that, articulations necessary to approach the problem in the country. Therefore, some of these actions include tracking, monitoring, data analysis on heat sources and health data in order to producing and sharing information to population, managers and stakeholders.
\end{abstract}

Keywords: air pollution, environmental health surveillance, fires. 